\title{
Geniculate neuralgia in an adolescent treated via sectioning of the nervus intermedius and microvascular decompression
}

\author{
Derek D. George, MS, ${ }^{1}$ and Thomas S. Ridder, MD² \\ 1 University of Colorado School of Medicine, Aurora; and 2UC Health Brain, Spine, and Physical Medicine and Rehabilitation \\ Clinic, Colorado Springs, Colorado
}

\begin{abstract}
Geniculate neuralgia or nervus intermedius (NI) neuralgia is a rare condition characterized by intermittent, severe, stabbing deep ear pain. The pain can be triggered by stimulation of the external ear and is sometimes accompanied by facial pain. The condition is thought to result, in part, from vascular compression of the NI, although other etiologies exist. To date, fewer than 150 cases have been described in the English-language literature, and only 1 case of surgically treated geniculate neuralgia with microvascular decompression (MVD) of cranial nerves VIII, IX, and X has been described in a pediatric patient. Here, the authors present the case of an adolescent boy with bilateral geniculate neuralgia treated at two different time points with sectioning of the NI and MVD.
\end{abstract}

https://thejns.org/doi/abs/10.3171/2020.3.PEDS19584

KEYWORDS geniculate neuralgia; nervus intermedius; microvascular decompression; adolescence; functional neurosurgery

$\mathrm{G}$ ENICUlate neuralgia (GN) or nervus intermedius (NI) neuralgia is a rare condition characterized by intermittent, severe, stabbing deep ear pain. Pain can be triggered by stimulation of the external ear and sometimes accompanied by facial pain. The condition is thought to result, in part, from vascular compression of the NI. To date, fewer than 150 cases have been described in the English-language literature, ${ }^{1}$ and only 1 case of GN treated with microvascular decompression (MVD) of cranial nerves (CNs) VIII, IX, and X has been described in a pediatric patient. ${ }^{2}$ Here, we present the case of an adolescent patient with bilateral GN treated at two different time points with sectioning of the NI and MVD.

\section{Case Report}

History

Here, we describe the case of a 17-year-old boy presenting with a 2-year history of bilateral, intermittent, stabbing deep ear pain rated $9 / 10$ on a numeric rating scale, primarily worse in the left ear. His pain was exacerbated by touch of the outer ear, wind on the outer ear, and changes in atmospheric pressure. His pain was accompanied by tinnitus.

\section{Examination}

Examination revealed significant tenderness to palpation of the left and right external auditory canal, but no cranial nerve motor deficits were noted. No tenderness to palpation or hyperalgesia was noted in the bilateral $\mathrm{CN} \mathrm{V}$ distributions.

\section{Workup}

High-resolution, multisequence MRI with and without contrast medium did not reveal obvious pathology or compression of cranial nerves on the right, but it did show possible vascular compression of CN VII on the left. Extensive workup with multiple specialties (pediatric neurology, pediatric and adult neurosurgery experts in functional/pain procedures, ENT, and anesthesia pain specialists) failed to elucidate alternative etiologies of otalgia. Specifically, ENT examination revealed no external auditory canal or tympanic membrane pathology, and the patient's audiogram was normal bilaterally. Considering his symptoms and workup did not suggest an alternative etiology, the diagnosis of left-sided GN was rendered. Medical management was first attempted: our patient was trialed on oxcarbazepine, $300 \mathrm{mg}$ twice daily for 1 month, but discontinued due to side effects. We then gave amitriptyline, $50 \mathrm{mg}$ daily, for

ABBREVIATIONS AICA = anterior inferior cerebellar artery; $\mathrm{CN}=$ cranial nerve; $\mathrm{GN}=$ geniculate neuralgia; $\mathrm{MVD}=$ microvascular decompression; $\mathrm{NI}=$ nervus intermedius; PTFE = polytetrafluorethylene. 

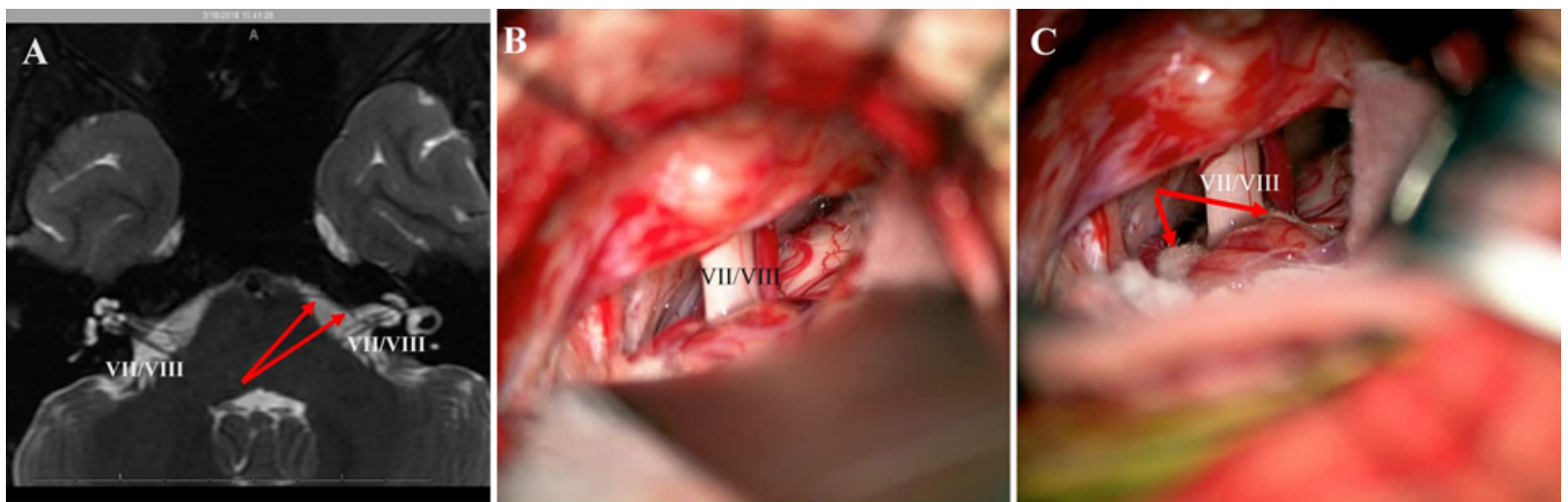

FIG. 1. Diagnostic and intraoperative images from the left-sided GN surgery. A: Axial T2-weighted, fat-suppressed MR image captured prior to the operation, showing CNs VII and VIII bilaterally at the cerebellopontine angle. A thin vascular loop of the AICA is shown anterior to CNs VII and VIII on the left (arrows). B: Intraoperative photograph showing the left AICA artery abutting CNs VII and VIII superiorly. C: Photograph of CNs VII and VIII after NI sectioning and MVD with PTFE placed superiorly and inferiorly (arrows). MR image captured using a Siemens GE T3 MRI scanner. Intraoperative images obtained with a Zeiss OPMI Pentero 900 surgical microscope. Figure is available in color online only.

7 months with no reported improvement in pain symptoms. Lastly, we tried gabapentin, $600 \mathrm{mg} 3$ times daily for 5 months, but he did not experience a decrease in pain symptoms. Due to the medically refractory symptoms of left ear pain and his overall refractory response to conservative measures, further discussion occurred with other pediatric and adult neurosurgeons familiar with the treatment of pain syndromes. It was collectively determined that surgical intervention was warranted. MVD of the left facial nerve with possible sectioning of the NI was planned as the first surgery.

\section{Operative Details}

For the left-sided surgery, neuromonitoring was used to ensure the safety of the cranial nerves. The patient was positioned in the right lateral decubitus position. A left retromastoid craniotomy was performed to access the posterior fossa. CNs V, VIII, IX, and X were visualized along the lateral brainstem. CN V was inspected, revealing no evidence of compression by vascular structures. Inspection of the vestibulocochlear complex revealed anatomical compression by a superior vascular loop and inferior vascular loop of the anterior inferior cerebellar artery (AICA). Both fascicles of the NI were identified near the root entry zone between CNs VII and VIII and were traced distally to the cisternal segment, where the NI was isolated and successfully sectioned. Polytetrafluorethylene (PTFE) was placed to separate the superior and inferior arterial loops from the vestibulocochlear complex (Fig. 1). A large vascular loop of AICA was also found to compress CNs IX and X inferiorly. This vascular loop was mobilized from the nerves, and PTFE was placed to separate the loop from these nerves. An autologous duraplasty utilizing pericranium was performed without signs of CSF leakage. No intraoperative complications were observed. Day 1 postoperatively, the patient reported significant improvement in left deep ear pain, and tactile stimulation of the external auditory canal did not elicit pain. No sensory loss, hearing loss, vertigo, loss of taste, or motor weakness was noted. At the initial 3-month follow-up visit, the patient no longer suffered from lancinating left ear pain that interfered with his daily functioning. At the 18-month follow-up visit, he no longer suffered from left ear pain or any other of his preoperative symptoms. No long-term complications were noted.

Unfortunately, the patient continued to suffer more generalized headaches and right-sided deep ear pain mirroring his left-sided GN symptoms. This chronic pain took a toll on our patient's mental health, and he was hospitalized with suicidal ideation and attempted suicide. Follow-up with a psychiatrist and pain management team was arranged. Due to the similarity of otalgia symptoms on the right side relative to the left, pain management and neurosurgery teams considered right-sided posterior fossa exploration for MVD and possible sectioning of the NI. After thorough discussion about surgical treatment options, the patient and his family elected to undergo right-sided severing of the NI with MVD of CNs VII, IX, and X.

Similar positioning, neuromonitoring, and approach were used for the right-sided surgery. Intraoperatively, we found the right labyrinthine artery wedged between the facial nerve and the vestibulocochlear complex, with the NI draped across the artery. This artery was successfully separated from the cranial nerves, and PTFE was placed between the nerves and the artery (Fig. 2). The NI was then identified and sectioned at the cisternal segment. We then turned to CNs IX and X inferiorly, finding a tortuous loop of the AICA compressing CN IX. PTFE was placed between the AICA loop and CN IX to decompress the nerve. No operative complications were observed, including vertigo, hearing loss, or facial weakness. At the initial 6-week follow-up visit, our patient reported complete resolution of his right-sided otalgia. At the 10-month follow-up visit, the patient no longer suffered from right-sided pain. He no longer expresses suicidal ideation or plans given his improvement in pain symptoms. No long-term complications were noted, including subjective taste loss.

\section{Discussion}

GN is a rare cranial neuralgia thought to involve the NI. The NI is a small nerve running with the motor por- 

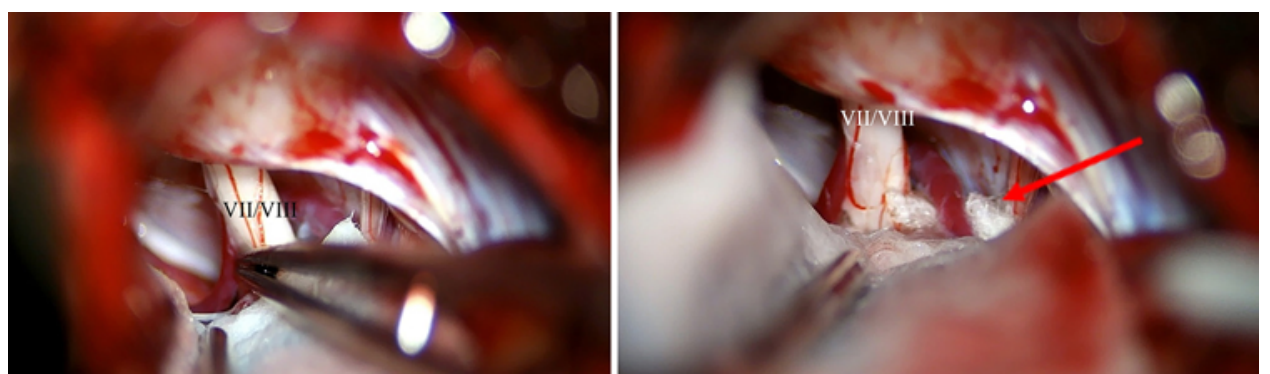

FIG. 2. Diagnostic and intraoperative images from the right-sided GN surgery. Left: Intraoperative photograph showing the right labyrinthine artery abutting CNs VII and VIII. Right: Photograph of CNs VII and VIII after NI sectioning and MVD with PTFE placed superiorly (arrow). Intraoperative images captured with a Zeiss OPMI Pentero 900 surgical microscope. Figure is available in color online only.

tion of the facial nerve. Its functions include the following: 1) taste innervation to the anterior two-thirds of the tongue via the chorda tympani nerve; 2) visceral efferent innervation of the lacrimal, sublingual, and submandibular glands, along with the mucous glands of the nasal and palatal mucosae; and 3) somatic innervation of a portion of the pinna and external auditory canal. Because of the diverse fiber composition of the NI, the symptomatology of GN is somewhat variable. The most common symptoms involve episodes of intense, stabbing deep ear pain. The pain may radiate to the retroauricular region, pinna, or soft palate ${ }^{1}$ and therefore can be associated with pain triggered by chewing and swallowing. ${ }^{2}$ Pain is often exacerbated by tactile stimulation of the external ear. In some cases, the neuralgia is associated with dysfunction of the visceral afferents and efferents contained in the nerve, affecting taste, lacrimation, and salivation. ${ }^{3}$ GN can sometimes co-occur with other cranial nerve neuralgias, making the diagnosis based on symptoms difficult. ${ }^{4}$

Between 1968 and 2013, around only 50 peer-reviewed reports had been published on $\mathrm{GN},{ }^{5}$ and fewer than 150 cases were reported in the English-language literature between 1932 and 2012. ${ }^{1}$ Due to the rarity of this condition, robust epidemiological data are unavailable. The etiology of GN is often vascular compression from vertebral artery branches, but similar lancinating pain can be caused by other intracranial pathology, ${ }^{6,7}$ as well as herpes zoster or neuroborreliosis infections ${ }^{8,9}$ Establishing the diagnosis of GN is often difficult, owing to multiple factors. The symptoms of paroxysmal ear pain can be attributed to various pathologies of the ear, as well as other cranial nerve neuralgias. Workup therefore requires multidisciplinary input from neurologists, neurosurgeons, dentists, audiologists, and otolaryngologists. ${ }^{1}$ Thin-slice MRI, possibly with MR angiography, is the best modality for evaluating vascular compression. ${ }^{10}$ However, imaging may sometimes show no obvious compression, ${ }^{11}$ as seen in our patient on his right side, and asymptomatic patients may show evidence of vascular compression. ${ }^{12}$ The contribution of imaging to the workup-while valuable-may be less sensitive and specific than desired.

The treatment of GN starts with pharmacological management. The most common pharmacological interventions are anticonvulsants such as carbamazepine and lamotrigine. Carbamazepine is often the first-line choice owning to its relatively high efficacy for the treatment of neuropathic pain. ${ }^{13}$ If the side effect burden is too great or the response is inadequate, lamotrigine is sometimes used. However, high-quality evidence for the use of lamotrigine in neuropathic pain is lacking..$^{14}$ Besides anticonvulsants, the tricyclic antidepressant amitriptyline is also used, with a minority of patients deriving benefit. ${ }^{15}$ Gabapentin is sometimes used, either as the first-line agent or after other medications have failed, ${ }^{8,16}$ and has moderate-quality evidence for its use in neuropathic pain. ${ }^{17}$ If pharmacological management fails, more invasive techniques can be utilized, such as peripheral nerve blocks. While no studies have investigated the efficacy of peripheral nerve blocks for the treatment of GN specifically, a systematic analysis of peripheral nerve block for neuralgia suggested a possible benefit. ${ }^{18}$

If medical management fails, surgery may be considered. Surgical treatment of GN most commonly involves sectioning of the NI but can involve excision of the geniculate ganglion or MVD of the NI at the root entry zone. In some cases, additional MVD of CNs V, IX, and $\mathrm{X}$ may be attempted. ${ }^{19}$ Data on the long-term efficacy of NI sectioning and MVD for GN have come from multiple institutional reviews. Lovely and Jannetta ${ }^{19}$ published a case series of 14 patients treated with MVD of CNs V, IX, and X with sectioning of the NI. They reported $90 \%$ $(9 / 10)$ of patients had partial or complete pain relief beyond the 12-month follow-up. A review by Peris-Celda et al. demonstrated favorable pain outcomes in $73 \%(8 / 11)$ of patients who underwent NI sectioning with or without MVD of CNs V and/or IX. ${ }^{4}$ Interestingly, a recent review by Holste et al. demonstrated that sectioning of the NI alone did not provide long-term pain relief, and patients with or without vascular compression experience similar pain-free survival periods. ${ }^{20}$ This suggests that the pathophysiology of GN is complex and may not involve just the NI but also CNs V and/or IX.

Here we have presented the case of bilateral GN in an adolescent boy treated via sectioning of the NI and MVD of CNs VIII and IX, with unilateral decompression of CN $\mathrm{X}$. To our knowledge, this is only the second case report detailing the treatment of GN via NI sectioning and MVD in a pediatric patient and may be the first case of such treatment bilaterally in an adolescent patient. The first case from Zenonos et al. ${ }^{2}$ described a 9-year-old boy who presented with a 1-year history of intermittent stabbing ear pain that was worsened by chewing and swallowing. 
Multidisciplinary workup failed to suggest an alternative etiology of otalgia, and the neuroleptic therapy failed to resolve the pain. MRI showed a vessel compressing CNs IX and $\mathrm{X}$ on the right, and NI sectioning with decompression of CNs IX and X was performed. At the 3-month followup visit, the patient reported complete resolution of pain. ${ }^{11}$ Rupa et al. ${ }^{7}$ and Holste et al. ${ }^{20}$ both included patients under 18 years of age in their reviews (a 12-year-old and a 13-year-old patient, respectively), but the mean patient age was 36.6 and 44.86 years, respectively, highlighting the rarity of GN in younger populations.

Our patient reported bilateral headaches with lancinating deep ear pain bilaterally of 2 years' duration, worse on the left than right. A multidisciplinary workup similarly failed to suggest an alternative etiology, including other cranial nerve neuralgias or Ramsay Hunt otalgia. In contrast to the case from Zenonos et al., ${ }^{2}$ imaging failed to suggest obvious vascular compression. Given the lack of alternative etiology, the diagnosis of GN was rendered. Our patient's pain was refractory to medical therapy, and he with his family elected to undergo surgery for left-sided GN without complications and with abatement of left-sided pain at a follow-up visit 18 months after surgery. Sadly, at around 5 months postoperatively, our patient was hospitalized for suicidal ideation related to his chronic rightsided otalgia. It was determined that his right-sided pain was likely due to vascular compression of the NI, and the patient and family elected to undergo right-sided posterior fossa exploration with a possible MVD and NI sectioning. Right-sided MVD of CN IX and sectioning of the NI were performed, and the patient experienced complete resolution of his right otalgia by the 10-month follow-up visit.

This patient's unusual presentation of bilateral GN highlights the enigmatic nature of chronic geniculate or atypical facial pain. Our case attests to the proactive use of MVD and/or rhizotomy for the treatment of GN, regardless of age, in appropriately selected patients. The study by Holste et al. ${ }^{20}$ of significant pain recurrence rates suggests that our patient may develop atypical facial pain in the future despite seemingly successful surgical intervention. Ultimately, further research into the epidemiology, complex pathophysiology of GN, and symptomatic overlap with other cranial neuralgias is needed to better guide successful treatment of this debilitating condition.

\section{References}

1. Tang IP, Freeman SR, Kontorinis G, et al. Geniculate neuralgia: a systematic review. J Laryngol Otol. 2014;128(5):394399.

2. Zenonos GA, Faraji A, Zwagerman NT, et al. A case report of pediatric geniculate neuralgia successfully treated with sectioning of the nervus intermedius and microvascular decompression of the cranial nerves IX and X. J Neurol Surg B. 2016;77:P071.

3. Sachs E Jr. The role of the nervus intermedius in facial neuralgia. Report of four cases with observations on the pathways for taste, lacrimation, and pain in the face. J Neurosurg. 1968;28(1):54-60.

4. Peris-Celda M, Oushy S, Perry A, et al. Nervus intermedius and the surgical management of geniculate neuralgia. $\mathrm{J} \mathrm{Neu-}$ rosurg. 2018;131(2):343-351.

5. Tubbs RS, Mosier KM, Cohen-Gadol AA. Geniculate neural- gia: clinical, radiologic, and intraoperative correlates. World Neurosurg. 2013;80(6):e353-e357.

6. Buyse GG, Caekebeke J, Demaerel P, Plets C. Primary brain stem tethering: a rare cause of geniculate neuralgia. J Laryngol Otol. 1999;113(10):945-947.

7. Rupa V, Saunders RL, Weider DJ. Geniculate neuralgia: the surgical management of primary otalgia. J Neurosurg. 1991;75(4):505-511.

8. Frese A, Lüttmann RJ, Husstedt IW, et al. Geniculate neuralgia as a manifestation of neuroborreliosis. Headache. 2002;42(8):826-828.

9. Tubbs RS, Steck DT, Mortazavi MM, Cohen-Gadol AA. The nervus intermedius: a review of its anatomy, function, pathology, and role in neurosurgery. World Neurosurg. 2013;79(56):763-767.

10. Yap L, Pothula VB, Lesser T. Microvascular decompression of cochleovestibular nerve. Eur Arch Otorhinolaryngol. 2008;265(8):861-869.

11. Chung SS, Chang JW, Kim SH, et al. Microvascular decompression of the facial nerve for the treatment of hemifacial spasm: preoperative magnetic resonance imaging related to clinical outcomes. Acta Neurochir (Wien). 2000;142(8):901907.

12. Saers SJ, Han KS, de Ru JA. Microvascular decompression may be an effective treatment for nervus intermedius neuralgia. J Laryngol Otol. 2011;125(5):520-522.

13. Wiffen PJ, Derry S, Moore RA, Kalso EA. Carbamazepine for chronic neuropathic pain and fibromyalgia in adults. $\mathrm{Co}$ chrane Database Syst Rev. 2014;4:CD005451.

14. Wiffen PJ, Derry S, Moore RA. Lamotrigine for chronic neuropathic pain and fibromyalgia in adults. Cochrane Database Syst Rev. 2013;12:CD006044.

15. Moore RA, Derry S, Aldington D, et al. Amitriptyline for neuropathic pain in adults. Cochrane Database Syst Rev. 2015;7:CD008242.

16. Magnus L. Nonepileptic uses of gabapentin. Epilepsia. 1999;40(suppl 6):S66-S74.

17. Wiffen PJ, Derry S, Bell RF, et al. Gabapentin for chronic neuropathic pain in adults. Cochrane Database Syst Rev. 2017;6:CD007938.

18. Vlassakov KV, Narang S, Kissin I. Local anesthetic blockade of peripheral nerves for treatment of neuralgias: systematic analysis. Anesth Analg. 2011;112(6):1487-1493.

19. Lovely TJ, Jannetta PJ. Surgical management of geniculate neuralgia. Am J Otol. 1997;18(4):512-517.

20. Holste KG, Hardaway FA, Raslan AM, Burchiel KJ. Painfree and pain-controlled survival after sectioning the nervus intermedius in nervus intermedius neuralgia: a single-institution review. J Neurosurg. 2018;131(2):352-359.

\section{Disclosures}

The authors report no conflict of interest concerning the materials or methods used in this study or the findings specified in this paper.

\section{Author Contributions}

Conception and design: both authors. Acquisition of data: both authors. Analysis and interpretation of data: both authors. Drafting the article: George. Critically revising the article: both authors. Reviewed submitted version of manuscript: both authors. Approved the final version of the manuscript on behalf of both authors: George. Administrative/technical/material support: both authors. Study supervision: Ridder.

\section{Correspondence}

Derek D. George: University of Colorado School of Medicine, Aurora, CO. derek.d.george@cuanschutz.edu. 